\title{
Prognostic significance of second mitochondria-derived activator of caspase (Smac/DIABLO) expression in bladder cancer and target for therapy
}

\author{
YOICHI MIZUTANI ${ }^{1}$, YOJI KATSUOKA ${ }^{1}$ and BENJAMIN BONAVIDA ${ }^{2}$ \\ ${ }^{1}$ Department of Urology, Osaka Medical College, Osaka 569-8686, Japan; ${ }^{2}$ Department of Microbiology, \\ Immunology and Molecular Genetics, Jonsson Comprehensive Cancer Center, UCLA School of \\ Medicine, University of California at Los Angeles, CA 90095, USA
}

Received March 22, 2010; Accepted May 17, 2010

DOI: 10.3892/ijo_00000699

\begin{abstract}
Although the expression of Smac/DIABLO has been reported in various cancers, little is known about its clinical significance in bladder cancer. The present study was designed to evaluate the relationship between progression of disease and Smac/DIABLO expression by clinical pathological analysis of patients with bladder cancer. The level of Smac/ DIABLO expression was quantified by Western blot analysis using non-fixed fresh frozen tissues derived from patients with bladder cancer. All normal bladders expressed Smac/ DIABLO. However, 64/84 (76\%) of bladder cancers expressed Smac/DIABLO and $24 \%$ were negative. In Ta and T1 superficial bladder cancers, 98\% expressed Smac/DIABLO, whereas only $41 \%$ expressed Smac/DIABLO in muscleinvasive bladder cancers. Smac/DIABLO expression inversely correlated with the grade of bladder cancer. Patients with Ta and T1 superficial bladder cancer with higher Smac/DIABLO expression had a longer postoperative recurrence-free period than those with lower Smac/DIABLO expression after transurethral resection in the 5-year follow-up. Patients with invasive bladder cancer expressing Smac/DIABLO had a longer postoperative disease-specific survival than those without Smac/DIABLO expression after radical cystectomy in the 5-year follow-up. The cisplatin-resistant T24 bladder cancer line (T24/CDDP) and the adriamycin-resistant T24
\end{abstract}

Correspondence to: Dr Yoichi Mizutani, Department of Urology, Osaka Medical College, 2-7 Daigakumachi, Takatsuki, Osaka 5698686, Japan

E-mail: uro046@poh.osaka-med.ac.jp

Abbreviations: ADR, adriamycin; CDDP, cisplatin; IAP, inhibitor of apoptosis protein; IBM, IAP-binding motif; MLS, mitochondrial leader sequence; Smac/DIABLO, second mitochondria-derived activator of caspase/direct inhibitor of apoptosis protein-binding protein with low pl; TUR-Bt, transurethral resection of bladder tumor; XIAP, X-linked inhibitor of apoptosis protein

Key words: Smac/DIABLO, bladder cancer, prognosis, apoptosis, cisplatin, adriamycin line (T24/ADR) showed lower level of Smac/DIABLO expression, compared with the T24 parental line. In conclusion, the present study demonstrates for the first time that Smac/ DIABLO expression was downregulated in bladder cancer, especially in high grade muscle-invasive bladder cancer, and that lower Smac/DIABLO expression in bladder cancer predicted a worse prognosis. In addition, the cisplatin-resistant T24/CDDP line and the adriamycin-resistant T24/ADR line expressed lower level of Smac/DIABLO expression. These results suggest that Smac/DIABLO expression in bladder cancer may be used as a prognostic parameter, and that low Smac/DIABLO expression in bladder cancer may be associated with resistance to chemotherapy.

\section{Introduction}

Bladder cancer accounts for approximately $4 \%$ of all cancer cases worldwide. Anticancer chemotherapy has demonstrated potential antitumor activity against bladder cancer $(1,2)$. However, the rate of complete response is low, and metastasis and recurrence still remain major problems in bladder cancer treatment.

Clinical factors have been used historically and clinically as prognostic markers such as stage and grade for patients with bladder cancer. Recent advances have been achieved in an attempt to understand the pathogenesis, behavior and molecular biology of bladder cancer with the objective of developing methods for early diagnosis, better prognosis and improved survival for patients with bladder cancer (3). Although numerous factors have been reported, few maintained independent significance in terms of overall survival (4). Moreover, several reported results have been controversial by studying the same biomarker. There are no identified molecular or cytogenetic tumor markers in bladder cancer that can help diagnosis, manage or confirm the remission, the progression or the relapse. Thus, there is an urgent need for the identification of new reliable diagnostic/prognostic markers and therapeutic targets in bladder cancer.

Tumor cells respond to cytotoxic therapies by activation of the type II mitochondrial apoptotic pathway. The activation of this pathway results in the depolarization of the mitochondrial membrane potential and the release of 
cytochrome c and second mitochondria-derived activator of caspase/direct inhibitor of apoptosis protein (IAP)-binding protein with low $\mathrm{p}$ (Smac/DIABLO) into the cytosol. These disrupt the apoptosome and lead to the activation of caspases 9, 8 and 3 (5). Smac was identified as a protein that can stimulate caspase 3 activation in cell extract (6). DIABLO was described independently by Verhagen et al (7). Smac/ DIABLO is expressed in most adult human tissues including heart, liver, pancreas, kidney, prostate and testis, at different levels. It is synthesized in the nucleus as a precursor protein of 239 amino acid residues and amino terminal mitochondrial leader sequence (MLS). Upon mitochondrial import, the MLS is removed by proteolysis, exposing the IAP-binding motif (IBM) at the N-terminus of mature Smac/DIABLO which is present as dimers. Through this IBM (Ala-Val-Pro-Ile), Smac/DIABLO is able to bind X-linked IAP (XIAP), cIAP-1 and cIAP-2 and survivin. One way by which Smac/DIABLO promotes caspase activation is by displacing caspase 9 coupled with XIAP (5). This inhibitory function of Smac/DIABLO relies on IBM. Thus, Smac/DIABLO's function is the inhibition of IAPs, and, therefore, Smac/DIABLO is considered as an apoptogenic factor.

Previous studies have reported that overexpression of Smac/DIABLO can induce apoptosis and/or sensitize the resistant cancer cells to death receptor- or cytotoxic agentinduced apoptosis $(8,9)$. These findings suggest that the level of Smac/DIABLO expression plays an important role in the regulation of apoptotic responses in cancer cells to both immune- and drug-mediated therapies. Therefore, we hypothesize that the levels of expression of Smac/DIALBO in bladder cancer may correlate with the progression of disease and may be of prognostic significance. There have been no reports on the expression of Smac/DIABLO in bladder cancers and its potential clinical significance. Thus, investigation of the level of Smac/DIABLO expression in bladder cancer was the focus of the present study. This study was designed to test our hypothesis and investigate the level of Smac/DIABLO expression in bladder cancer and determine its prognostic significance.

\section{Materials and methods}

Patients. Surgical specimens were obtained from 84 patients with bladder cancer. They included 75 male and 9 female patients, ranging in age from 31 to 87 years. Histological diagnosis revealed that all patients had urothelial carcinoma and none of the patient had metastasis. According to the TNM classification (UICC, 6th edition, 2002) the bladder cancers were: Ta $(n=26), T 1 \quad(n=26), T 2 \quad(n=15), T 3(n=13)$, T4 $(n=4)$, and G1 $(n=9), G 2(n=53)$, and G3 $(n=22)$. The specimens of bladder cancers and normal bladders were obtained at operation. Normal bladders $(n=25)$ were derived from the invasive bladder cancer patients undergoing radical cystectomy. The specimens were stored frozen at $-80^{\circ} \mathrm{C}$ until use for the assay of Smac/ DIABLO expression. The patients had no treatments for bladder cancer before surgery. This study was performed after approval by a local Human Investigations Committee. Informed consent was obtained from each patient.

Patients with Ta and T1 superficial bladder cancer were not treated after transurethral resection of bladder tumor

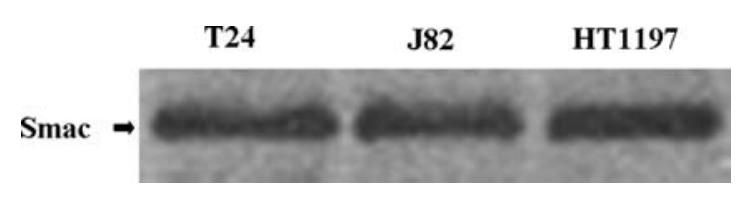

Figure 1. Expression of Smac/DIABLO in T24, J82, HT1197 bladder cancer cell lines. Smac/DIABLO expression in T24, J82, HT1197 bladder cancer cell lines was examined by Western blot analysis as described in Materials and methods.

(TUR-Bt) until recurrence. When patients with invasive bladder cancer had recurrence or metastasis after radical cystectomy, they were treated with cisplatin (CDDP)-based chemotherapy.

Reagents. CDDP and adriamycin (ADR) were kindly supplied by Nippon Kayaku Co. Ltd., Tokyo, Japan, and Kyowa Hakkou Kirin Co. Ltd, Tokyo, Japan, respectively.

Bladder cancer cell lines. T24, J82 and HT1197 human bladder cancer cell lines $(10,11)$ were maintained in monolayers on plastic dishes in RPMI-1640 medium (Gibco, Bio-cult, Glasgow, Scotland, UK) supplemented with 25 mM HEPES (Gibco), 2 mM L-glutamine (Gibco), 1\% non-essential amino acid (Gibco), $100 \mathrm{U} / \mathrm{ml}$ penicillin (Gibco), $100 \mu \mathrm{g} / \mathrm{ml}$ streptomycin (Gibco) and 10\% heat-inactivated fetal bovine serum (Gibco), hereafter referred to as complete medium. T24/CDDP and T24/ADR bladder cancer cell lines were CDDP-resistant and ADR-resistant T24 cell lines, respectively $(12,13)$.

Measurement of Smac/DIABLO expression in bladder cancer and normal bladder by Western blot analysis and definition of Smac/DIABLO expression. The expression of Smac/ DIABLO was determined by Western blot analysis as previously described $(14,15)$. Tumor and normal tissues were prepared to generate lysates and the protein concentration was determined. Twenty micrograms of the sample proteins were electrophoresed on $7.5 \%$ polyacrylamide gels in Trisglycin buffer and transferred to nitrocellulose membranes. The membrane was blocked for $30 \mathrm{~min}$ in blocking buffer (5\% skim milk in 1\% Tween-PBS ) and probed first with the rabbit anti-Smac/DIABLO antibody (Imgenex, San Diego, CA) for $1 \mathrm{~h}$. The membrane was washed and then incubated with peroxidase-conjugated goat anti-rabbit $\operatorname{IgG}$ and developed with the use of an enhanced chemiluminescence detection kit (Amersham Pharmacia Biotech, Piscataway, NJ). The relative expression of Smac/DIABLO protein was determined with a chemiluminescence imaging system and quantified by image analysis (Gel Doc 2000: Bio-Rad, Osaka, Japan).

The T24 bladder cancer cell line constitutively expressed Smac/DIABLO and was used as the internal standard to compare assays. All samples were analyzed at the same time. Repeated measurements yielded the same results. When Smac/DIABLO expression was not visually observed by the Western blot analysis, it was regarded as negative expression. In contrast, Smac/DIABLO expression was regarded as positive expression if a visual band was detected by Western blot analysis regardless of the variation of the levels of expression. Positive expression meant unambiguous visual 
A

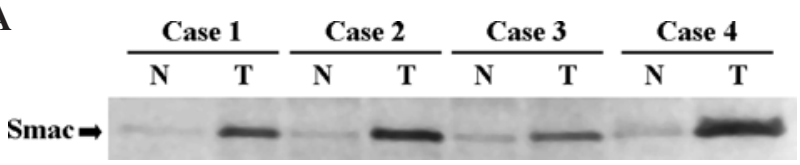

B

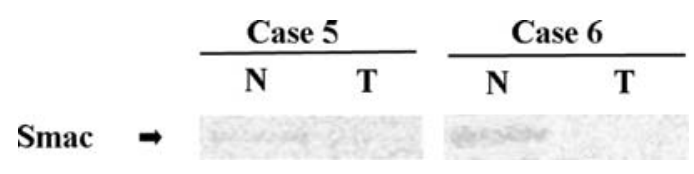

Figure 2. Expression of Smac/DIABLO in bladder cancer and normal bladder. (A) Smac/DIABLO expression in 4 cases of bladder cancer and corresponding normal bladder was examined by Western blot analysis as described in Materials and methods. The bladder cancers showed positive Smac/DIABLO expression. (B) Smac/DIABLO expression in 2 cases of bladder cancer and corresponding normal bladder was examined by Western blot analysis as described in Materials and methods. The bladder cancers showed negative Smac/DIABLO expression. N, normal bladder; T, bladder cancer.

Table I. Frequency of Smac/DIABLO expression in bladder cancer and normal bladder.

\begin{tabular}{lccc}
\hline $\begin{array}{l}\text { Bladder cancer and } \\
\text { normal bladder }\end{array}$ & $\begin{array}{l}\text { Smac/DIABLO expression }(\%)^{\mathrm{a}} \\
\text { Positive }\end{array}$ & $\begin{array}{c}\text { Ratio of Smac/DIABLO expression level to } \\
\text { mean level of normal bladder (mean } \pm \text { SE) }\end{array}$ \\
\hline $\begin{array}{l}\text { Normal bladder }(\mathrm{n}=25)^{\mathrm{b}} \\
\text { Bladder cancer }(\mathrm{n}=84)\end{array}$ & $25(100)$ & $0(0)$ & $5.06 \pm 0.44^{\mathrm{e}}$ \\
T-stage & $64(76)$ & $20(24)$ & $8.34 \pm 0.61$ \\
Ta $(\mathrm{n}=26)$ & $26(100)$ & $0(0)$ & $6.12 \pm 0.67^{\mathrm{f}}$ \\
T1 $(\mathrm{n}=26)$ & $25(96)$ & $1(4)$ & $7.23 \pm 0.44$ \\
Ta + T1 $(\mathrm{n}=52)^{\mathrm{c}}$ & $51(98)$ & $19(2)$ & $1.56 \pm 0.44^{\mathrm{g}}$ \\
T2 - T4 $(\mathrm{n}=32)$ & $13(41)$ & & $8.51 \pm 0.83$ \\
Tumor grade & & $0(0)$ & $6.06 \pm 0.56^{\mathrm{h}}$ \\
Grade $1(\mathrm{n}=9)$ & $9(100)$ & $6(11)$ & $1.33 \pm 0.44^{\mathrm{h}, \mathrm{i}}$ \\
Grade 2 $(\mathrm{n}=53)$ & $47(89)$ & $14(64)$ & \\
Grade $3(\mathrm{n}=22)$ & $8(36)$ & & \\
\hline
\end{tabular}

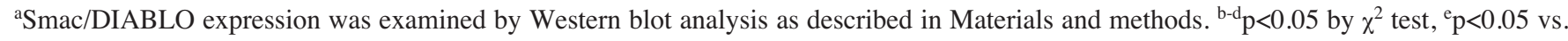
normal bladder, ${ }^{\mathrm{f}} \mathrm{p}<0.05$ vs. Ta, ${ }^{\mathrm{g}} \mathrm{p}<0.05$ vs. Ta $+\mathrm{T} 1,{ }^{\mathrm{h}} \mathrm{p}<0.05$ vs. Grade $1,{ }^{\mathrm{i}} \mathrm{p}<0.05$ vs. Grade 2 .

detection of Smac/DIABLO protein band by chemiluminescence and did not refer to the level of Smac/DIABLO expression. Based on the analysis of postoperative recurrencefree rate after TUR-Bt in patients with Ta and T1 superficial bladder cancer, the level of Smac/DIABLO expression greater than the median value was regarded as higher expression and the level of Smac/DIABLO expression less than the median value was regarded as lower expression.

Statistical analysis. For statistical analysis, Student's t-test and $\chi^{2}$ test were used. Postoperative recurrence-free rate and diseasespecific survival were determined by the Kaplan-Meier method. The Cox-Mantel test was used to establish the statistical difference in recurrence and survival. A $\mathrm{p} \leq 0.05$ was considered significant.

\section{Results}

Expression of Smac/DIABLO in bladder cancer cell lines, bladder cancers and normal bladders. The levels of Smac/ DIABLO in cell lysates of bladder cancer cell lines, bladder cancers and normal bladders were determined by Western blot analysis as described in Materials and methods. The T24, J82 and HT1197 bladder cancer cell lines expressed similar levels of Smac/DIABLO (Fig. 1).
Smac/DIABLO expression was determined in 25 normal bladders and 84 bladder cancers. Representative data of Smac/DIABLO expression of bladder cancer and normal bladders from the same patients are shown in Fig. 2A and B. The levels of Smac/DIABLO in normal bladders were very low when compared to the levels in bladder cancers. The expression levels of Smac/DIABLO in bladder cancers varied as a function of the stage of disease. In some patients (cases no. 1-4: Case 1: T1, G3, Case 2: T3, G2, Case 3: T2, G3, Case 4: T2, G3), the level of Smac/DIABLO expression in bladder cancers was higher than that in normal bladders (Fig. 2A). However, Smac/DIABLO expression was not detected in bladder cancers of cases 5 and 6 (Case 5: T4, G2, Case 6: T3, G3) (Fig. 2B).

The percentages of cases expressing Smac/DIABLO and those not expressing Smac/DIABLO were determined and summarized in Table I. Smac/DIABLO expression was detected in all normal bladder specimens. Overall, 64 (76\%) bladder cancers were positive for Smac/DIABLO and 20 (24\%) were negative. In Ta and T1 superficial bladder cancer $(\mathrm{n}=52), 51(98 \%)$ were positive and only one $(2 \%)$ was negative. In contrast, $13(41 \%)$ were positive and $19(59 \%)$ were negative in T2-T4 muscle-invasive bladder cancer $(n=32)$. The ratio of Smac/DIABLO expression in superficial bladder cancer compared to mean level of normal bladder 


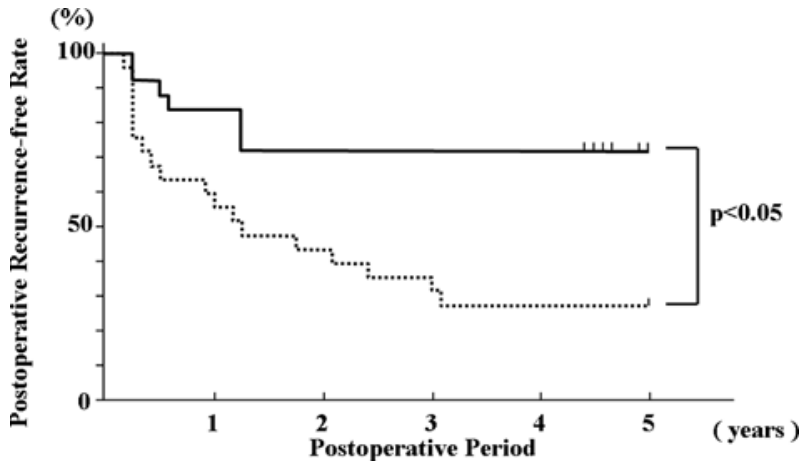

Figure 3. Relationship between Smac/DIABLO expression and postoperative recurrence-free rate after TUR-Bt in patients with Ta and T1 superficial bladder cancer. Postoperative tumor-free period of Ta and T1 superficial bladder cancer patients undergoing transurethral resection was determined by the Kaplan-Meier method. The level of Smac/DIABLO expression greater than the median value was regarded as higher expression and the level of Smac/DIABLO expression less than the median value was regarded as lower level. There was a significant difference in tumor-free interval between the following two groups in the 5-year follow-up $(\mathrm{P}<0.01$ by Cox-Mantel test). — , 25 patients with higher Smac/DIABLO expression; ----, 25 patients with lower Smac/DIABLO expression.

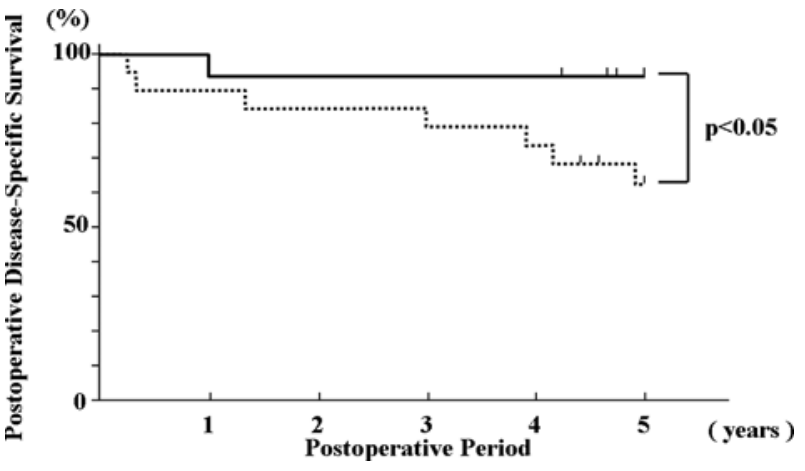

Figure 4. Relationship between Smac/DIABLO expression and postoperative disease-specific survival after radical cystectomy in patients with invasive bladder cancer. Postoperative clinical course of invasive bladder cancer patients undergoing radical cystectomy was determined by the Kaplan-Meier method. When Smac/DIABLO expression was not visually observed by the Western blot analysis, it was regarded as negative expression. In contrast, expression of Smac/DIABLO was regarded as positive expres-sion, if a visual band was detected by Western blot analysis. There was a significant difference in disease-specific survival rate between the following two groups in the 5 -year follow-up $(\mathrm{P}<0.05$ by Cox-Mantel test). -- 15 patients with positive Smac/DIABLO expression; ----, 19 patients with negative Smac/DIABLO expression.
A

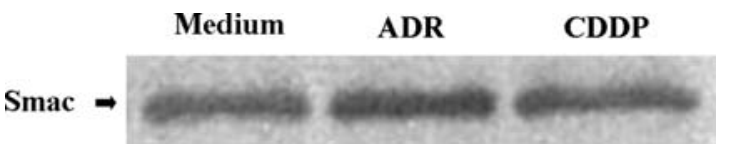

B

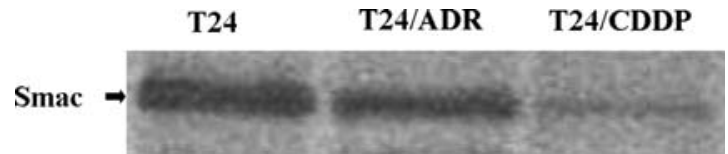

Figure 5. (A) Smac/DIABLO expression in T24 cells treated with ADR or CDDP Smac/DIABLO expression in T24 cells treated with ADR (0.1 $\mu$ g/ml, 3 h) or CDDP $(0.1 \mu \mathrm{g} / \mathrm{ml}, 3 \mathrm{~h})$ was examined by Western blot analysis as described in Materials and methods. (B) Smac/DIABLO expression in T24, T24/ADR, T24/CDDP bladder cancer cell lines Smac/DIABLO expression in T24, T24/ADR, T24/CDDP bladder cancer cell lines was examined by Western blot analysis as described in Materials and methods.

was 7.23, and that in muscle-invasive bladder cancer was 1.56. In addition, the ratio of Smac/DIABLO expression in Ta bladder cancer compared to normal bladder was 8.34 , and that in T1 bladder cancer was 6.12. These findings were corroborated with analysis of the grade of bladder cancer. In Grade 1/2 bladder cancer ( $\mathrm{n}=62), 56(90 \%)$ were positive and $6(10 \%)$ were negative. In contrast, in Grade 3 bladder cancer $(\mathrm{n}=22), 8(36 \%)$ were positive and $14(64 \%)$ were negative. The ratio of Smac/DIABLO expression in Grade 1, Grade 2 and Grade 3 bladder cancers compared to normal baldder was $8.51,6.06$ and 1.33 , respectively. These data show that the level of Smac/DIABLO expression inversely correlated with both the stage of disease progression and the increase of the histological grade of bladder cancer.

Correlation between Smac/DIABLO expression and postoperative specific survival in patients with bladder cancer. Patients with bladder cancer were evaluated for the postoperative clinical course. Postoperative recurrence-free rate and disease-specific survival were estimated by Kaplan-Meier analysis.

Based on the analysis of postoperative recurrence-free rate after TUR-Bt, patients with Ta and T1 superficial bladder cancer were divided into two groups, namely, those with higher Smac/DIABLO expression and those with lower expression as described in the Materials and methods section. Patients with superficial bladder cancer with higher Smac/DIABLO expression had a longer postoperative recurrence-free period, when compared to patients with lower expression of Smac/ DIABLO in the 5-year follow-up (Fig. 3).

Based on the analysis of postoperative disease-specific survival after radical cystectomy, patients with invasive bladder cancer were divided into two groups, namely, those with positive Smac/DIABLO expression and those with negative expression as described in Materials and methods section. Patients with invasive bladder cancer with positive Smac/ DIABLO expression had a longer disease-specific survival, when compared to patients with negative expression of Smac/DIABLO in the 5-year follow-up (Fig. 4).

These findings suggest that the level of Smac/DIABLO expression in bladder cancer may be a prognostic indicator, and that higher Smac/DIABLO expression in bladder cancer may be a good prognostic sign.

Drug-resistant bladder cancer cell lines express low levels of Smac/DIABLO compared to levels in parental cell lines. It has been reported that Smac/DIABLO is associated with drugsensitivity in cancer cells $(8,14,16)$. We, thus, examined the effect of CDDP and ADR, which are used for bladder cancer clinically, on the expression of Smac/DIABLO in bladder cancer. When the T24 bladder cancer cell line was treated with CDDP at concentrations of $0.1-10 \mu \mathrm{g} / \mathrm{ml}$ for different time 
periods of 3 or $12 \mathrm{~h}$, Smac/DIABLO expression did not change (Fig. 5A). Furthermore, when J82 and HT1197 bladder cancer cell lines were used as targets, similar results were obtained. ADR also had no effect on the expression of Smac/DIABLO in these bladder cancer cell lines (Fig. 5A).

The T24/CDDP and T24/ADR bladder cancer cell lines are CDDP-resistant and ADR-resistant T24 sublines, respectively $(12,13)$. The expression of Smac/DIABLO in T24/ CDDP and T24/ADR cell lines was lower, when compared with the expression of Smac/DIABLO in the T24 parental line (the ratios of Smac/DIABLO expression level compared to the T24 parental line were 0.21 and 0.63 , respectively) (Fig. 5B). These findings suggest that low expression of Smac/DIABLO in bladder cancer may be associated with drug-resistance.

\section{Discussion}

The present study provides evidence for the first time that the level of Smac/DIABLO expression inversely correlates with both the progression of the stage and the increase of the grade of bladder cancer. Patients with Ta and T1 superficial bladder cancers with higher Smac/DIABLO expression had a longer postoperative recurrence-free period than those with lower Smac/DIABLO expression in the 5-year follow-up. Furthermore, patients with invasive bladder cancer with positive Smac/DIABLO expression had a longer postoperative disease-specific survival than those with negative Smac/ DIABLO expression in the 5-year follow-up. The findings suggest that the level of Smac/DIABLO in bladder cancer may play an important role in regulating the tumor cell response to cytotoxic therapies and may be of prognostic significance in bladder cancer.

This study has demonstrated that approximately $24 \%$ of bladder cancer had no detectable Smac/DIABLO expression, although all normal bladder specimens expressed low levels of Smac/DIABLO. Moreover, advanced high grade diseases had lower expression of Smac/DIABLO than those with low stage/grade diseases. A recent study by Yoo et al has reported the analysis of archival tissues of carcinoma and sarcoma by immunohistochemical analysis for the expression of Smac/ DIABLO (17). Smac/DIABLO expression was observed in $62 \%$ of carcinoma and $22 \%$ of sarcoma. The level of Smac/ DIABLO expression varied depending on the individual tumor that was evaluated. For instance, 24 out of 42 gastric adenocarcinoma were positive for Smac/DIABLO whereas the remaining 18 were negative. Another study corroborated these data (18). Our previous study and those of others showed that normal kidney tissues adjacent to the cancer showed higher levels of Smac/DIABLO expression compared to cancer tissues and that progression of renal cell carcinoma was associated with low level of Smac/DIABLO expression $(14,19)$. Similar results were obtained in other cancers including colorectal cancer and lung cancer $(20,21)$. Our studies and those of others demonstrate that the expression level of Smac/DIABLO in carcinoma and sarcoma is significantly different from the corresponding non-cancerous tissues and correlate with cancer progression. Thus, the findings suggest strongly that the expression level of Smac/DIABLO might play an important role in the pathogenesis of cancer and cancer progression.
The current study demonstrates for the first time that Smac/DIABLO expression in bladder cancer predicted the clinical outcome. Similar results were reported with other cancers $(14,21,22)$. The precise underlying mechanism responsible for this relationship remains unclear. Since Smac/ DIABLO is a proapoptotic regulatory molecule, it is reasonable to assume that in spite of treatments, clones of cells which do not express Smac/DIABLO will not undergo apoptosis and will be selected to grow more easily and rapidly than clones which overexpress Smac/DIABLO. Thus, the present findings suggest that Smac/ DIABLO mimetics may provide a therapeutic means of preventing the growth of bladder cancer.

Cytotoxic chemotherapy, an integral part of the therapeutic approach for many solid tumors, has shown potential antitumor activity against bladder cancer $(23,24)$. However, the durable complete response rate is low and both metastasis and recurrence remain major problems in the therapy for bladder cancer. Therefore, new therapeutic approaches are required. Smac/DIABLO is a proapoptotic mitochondrial protein that is released to the cytosol in the cells in response to apoptotic stimuli including chemotherapeutic agents $(8,16)$. Our observation that Smac/DIABLO expression was downregulated in CDDP-resistant and ADR-resistant bladder cancer cell line, as compared to levels in wild-type, may be clinically relevant in the management of patients with bladder cancer. The endogenous low level of Smac/DIABLO in CDDP-resistant and ADR-resistant bladder cancer cells may not be adequate to neutralize the anti-apoptotic mechanisms regulated by IAPs. Therefore, chemotherapy in combination with Smac/DIABLO mimetics including small molecules that mimic Smac/DIABLO functions may be a promising treatment strategy against bladder cancer. For instance, Arnt et al reported that the first four amino acids of Smac/ DIABLO increased apoptosis in cell lines treated with paclitaxel, etoposide, camptothecin, and ADR (16).

IAPs such as XIAP are highly expressed in various cancers and are associated with poor prognosis and resistance to apoptosis $(25,26)$. Preliminary experiments demonstrated that the expression of XIAP was higher in high stage and high grade bladder cancers. Since XIAP blocks apoptosis at the effector phase, strategies targeting XIAP as well as Smac/ DIABLO may also be effective to overcome resistance to apoptosis. Smac/DIABLO is able to bind to IAP family members and XIAP is predominantly a Smac/DIABLObinding protein. Smac/DIABLO binds to XIAP, displaces XIAP from caspase-9, promotes cleavage of effector caspases and induces apoptosis $(27,28)$. In addition, Smac/DIABLO is also regulated by IAP family members. IAP family members including XIAP induce the ubiquitination and degradation of Smac/DIABLO (29,30). Furthermore, Smac3, a Smac/ DIABLO isoform, was recently identified (31). Smac3 induces the auto-ubiquitination and degradation of XIAP. Thus, the balance of IAP family and Smac/DIABLO may be important to maintain for the response to therapy. Further studies are therefore needed to determine the regulatory effects of Smac/ DIABLO expression in bladder cancers. The measurement of IAP family expression as well as Smac/DIABLO expression may be necessary for the accurate evaluation of the prognostic value of Smac/DIABLO and the efficacy of therapy with Smac/DIABLO mimetics. 
In conclusion, the current study demonstrates that Smac/ DIABLO expression was downregulated in high grade advanced bladder cancer, and that lower Smac/DIABLO expression was a negative prognostic sign. These findings suggest that the assessment of Smac/DIABLO expression may be useful in the management of bladder cancer. Since Smac/ DIABLO expression could be used as a prognostic parameter in patients with bladder cancer, the accurate prediction of prognosis may help select patients for more intensive surgical or chemotherapeutic approaches in combination with Smac/ DIABLO agonists. In addition, the potential biological significance of Smac/DIABLO expression as a component of a more generalized problem of apoptotic resistance requires further investigation and warrants future clinical studies.

\section{Acknowledgements}

This study was supported in part by Grant-in-Aids from the Japanese Ministry of Education, Culture, Sports, Science and Technology (no. 18390430).

\section{References}

1. Sternberg CN, Yagoda A and Scher HI: Preliminary results of M-VAC (methotrexate, vinblastine, doxorubicin and cisplatin) for transitional cell carcinoma of the urothelium. J Urol 133: 403-407, 1985.

2. Von der Maase H, Hansen SW and Roberts JT: Gemcitabine and cisplatin versus methotrexate, vinblastine, doxorubicin and cisplatin in advanced or metastatic bladder cancer: results of a large, randomized, multinational, multicenter, phase III study. J Clin Oncol 17: 3068-3077, 2000.

3. Grossman HB, Soloway M and Messing E: Surveillance for recurrent bladder cancer using a point-of-care proteomic assay. JAMA 295: 299-305, 2007

4. Bajorin DF, Dodd PM and Mazumdar M: Long-term survival in metastatic transitional-cell carcinoma and prognostic factors predicting outcome of therapy. J Clin Oncol 17: 3173-3181, 1999

5. Srinivasula SM, Datta P, Fan XJ, Fernandes-Alnemri T, Huang Z and Alnemri ES: Molecular determinants of the caspase-promoting activity of Smac/DIABLO and its role in the death receptor pathway. J Biol Chem 275: 36152-36157, 2000.

6. Du C, Fang M, Li Y, Li L and Wang X: Smac, a mitochondrial protein that promotes cytochrome c-dependent caspase activation by eliminating IAP inhibition. Cell 102: 33-42, 2000.

7. Verhagen AM, Ekert PG, Pakusch M, Silke J, Connolly LM, Reid GE, Moritz RL, Simpson RJ and Vaux DL: Identification of DIABLO, a mammalian protein that promotes apoptosis by binding to and antagonizing IAP proteins. Cell 102: 43-53, 2000.

8. Fulda S, Wick W, Weller M and Debatin KM: Smac agonists sensitize for Apo2L/TRAIL- or anticancer drug-induced apoptosis and induce regression of malignant glioma in vivo. Nat Med 8: 808-815, 2002.

9. Ng CP and Bonavida B: X-linked inhibitor of apoptosis (XIAP) blocks Apo-2 ligand/tumor necrosis factor-related apoptosisinducing ligand-mediated apoptosis of prostate cancer cells in the presence of mitochondrial activation: Sensitization by overexpression of second mitochondria-derived activator of caspase/ direct IAP-binding protein with low pl (Smac/DIABLO). Mol Cancer Ther 1: 1051-1058, 2002.

10. Mizutani Y, Matsubara H, Yamamoto K, Li YN, Mikami K, Okihara K, Kawauchi A, Bonavida B and Miki T: Prognostic significance of serum osteoprotegerin levels in patients with bladder cancer. Cancer 101: 1794-1802, 2004.

11. Mizutani Y, Wada H, Fukushima M, Yoshida O, Nakanishi H, Li YN and Miki T: Prognostic significance of orotate phosphoribosyltransferase activity in bladder cancer. Cancer 100: 723-731, 2004.
12. Mizutani Y, Nakanishi H, Li YN, Sato N, Kawauchi A and Miki T: Enhanced sensitivity of bladder cancer cells to cisplatinmediated cytotoxicity and apoptosis in vitro and in vivo by the selective cyclooxygenase-2 inhibitor JTE-522. J Urol 172: $1474-1479,2004$

13. Mizutani Y, Yoshida O, Miki T and Bonavida B: Synergistic cytotoxicity and apoptosis by Apo-2 ligand and adriamycin against bladder cancer cells. Clin Cancer Res 5: 2605-2612, 1999.

14. Mizutani Y, Nakanishi H, Yamamoto K, Li YN, Matsubara H, Mikami K, Okihara K, Kawauchi A, Bonavida B and Miki T: Downregulation of Smac/DIABLO expression in renal cell carcinoma and its prognostic significance. J Clin Oncol 23: 448-454, 2005

15. Mizutani Y, Kawauchi A, Benjamin B and Miki T: Smac/ DIABLO: A proapoptotic molecular target in renal cell cancer. In: Renal Cell Carcinoma: Molecular Targets and Clinical Application. Bukowski RM, Figlin RA and Motzer RJ (ed). The Humana Pres Inc., New Jersey, pp335-346, 2009.

16. Arnt CR, Chiorean MV, Heldebrant MP, Gores GJ and Kaufmann SH: Synthetic Smac/DIABLO peptides enhance the effects of chemotherapeutic agents by binding XIAP and cIAP1 in situ. J Biol Chem 277: 44236-44243, 2002.

17. Yoo NJ, Kim HS, Kim SY, Park CH, Jeon HM, Jung ES, Lee JY and Lee SH: Immunohistochemical analysis of Smac/DIABLO expression in human carcinomas and sarcomas. APMIS 111: 382-388, 2003.

18. Shibata T, Mahocka C, Wethkamp N, Heikaus S, Gabbert HE and Ramp U: Disturbed expression of the apoptosis regulators XIAP, XAF1, and Smac/DIABLO in gastric adenocarcinomas. Diagn Mol Pathol 16: 1-8, 2007.

19. Kempkensteffen C, Hinz S, Christoph F, Krause H, Magheli A, Schrader M, Schostak M, Miller K and Wreikert S: Expression levels of the mitochondrial IAP antagonists Smac/DIABLO and $\mathrm{Omi} / \mathrm{HtrA} 2$ in clear-cell renal cell carcinomas and their prognostic value. J Cancer Res Clin Oncol 134: 543-550, 2008.

20. Endo K, Kohnoe S, Watanabe A, Tashiro H, Sakata H, Morita M, Kakeji Y and Maehara Y: Clinical significance of Smac/ DIABLO expression in colorectal cancer. Oncol Rep 21: 351-355, 2009.

21. Sekimura A, Konishi A, Mizuno K, Kobayashi Y, Sasaki H, Yano M, Fukai I and Fujii Y: Expression of Smac/DIABLO is a novel prognostic marker in lung cancer. Oncol Rep 11: 797-802, 2004.

22. Bao ST, Gui SQ and Lin MS: Relationship between expression of Smac and Survivin and apoptosis of primary hepatocellular carcinoma. Hepatobiliary Pancreat Dis Int 5: 580-583, 2006.

23. Loehrer PJ, Einhorn LH and Elson PJ: A randomized comparison of cisplatin alone or in combination with methotrexate, vinblastine, and doxorubicin in patients with metastatic urothelial carcinoma: a cooperative group study. J Clin Oncol 10: 1066-1073, 1992.

24. Von der Maase H, Sengelov L and Roberts JT: Long-term survival results of a randomized trial comparing gemcitabine plus cisplatin, with methotrexate, vinblastine, doxorubicin, plus cisplatin in patients with bladder cancer. J Clin Oncol 23: 4602-4608, 2005

25. Deveraux QL and Reed JC: IAP family proteins - suppressors of apoptosis. Genes Dev 13: 239-252, 1999.

26. Sasaki H, Sheng Y, Kotsuji F and Tsang BK: Down-regulation of X-linked inhibitor of apoptosis protein induces apoptosis in chemoresistant human ovarian cancer cells. Cancer Res 60: 5659-5666, 2000

27. Goyal L: Cell death inhibition: Keeping caspases in check. Cell 104: 805-808, 2001.

28. Srinivasula SM, Hegde R, Saleh A, Datta P, Shiozaki E, Chai J, Lee RA, Robbins PD, Fermandes-Alnemri T, Shi Y and Alnemri ES: A conserved XIAP-interaction motif in caspase-9 and Smac/DIABLO regulates caspase activity and apoptosis. Nature 410: 112-116, 2001.

29. Morizane Y, Honda R, Fukami K and Yasuda H: X-linked inhibitor of apoptosis function as ubiquitin ligase toward mature caspase-9 and cytosolic Smac/DIABLO. J Biochem 137: 125-132, 2005.

30. Hu S and Yang X: Cellular inhibitor of apoptosis 1 and 2 are ubiquitin ligases for the apoptosis inducer Smac/DIABLO. J Biol Chem 278: 10055-10060, 2003.

31. Fu J, Jin Y and Arend LJ: Smac3, a novel Smac/DIABLO splicing variant, attenuates the stability and apoptosis-inhibiting activity of X-linked inhibitor of apoptosis protein. J Biol Chem 278: 52660-52672, 2003. 